\title{
Exploring the behavioral reactions to a mirror in the nocturnal grey mouse lemur: sex differences in avoidance
}

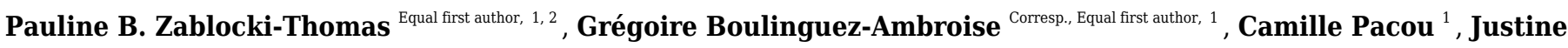 \\ Mézier $^{1}$, Anthony Herrel ${ }^{1,3}$, Fabienne Aujard ${ }^{1}$, Emmanuelle Pouydebat ${ }^{1}$ \\ ${ }^{1}$ UMR 7179, Département d'Écologie et de Gestion de la Biodiversité, Museum national d'Histoire naturelle, PARIS, France \\ 2 California National Primate Research Center, Davis, California, United States of America \\ 3 Evolutionary Morphology of Vertebrates, Ghent University, Ghent, Belgium \\ Corresponding Author: Grégoire Boulinguez-Ambroise \\ Email address: gregoire.boulinguez-ambroise@cri-paris.org
}

Most mirror-image stimulation studies (MIS) have been conducted on social and diurnal animals in order to explore self-recognition, social responses, and personality traits. Small, nocturnal mammals are difficult to study in the wild and are under-represented in experimental behavioral studies. In this pilot study, we explored the behavioral reaction of a small nocturnal solitary forager - the grey mouse lemur (Microcebus murinus) - an emergent animal model in captivity. We assessed whether MIS can be used to detect a repeatable behavioral reaction, whether individuals will present a similar reaction toward a conspecific and the mirror, and whether males and females respond similarly. We tested 12 individuals ( 6 males and 6 females) twice in three different contexts: with a mirror, with a live conspecific, and with a white board as a neutral control. We detected significant repeatability for the activity component of the behavioral reaction. There was a significant effect of the context and the interaction between presentation context and sex for avoidance during the first session for males but not for females. Males avoided the mirror more than they avoided a live conspecific. This pilot study opens a discussion on the behavioral differences between males and females regarding social interactions and reproduction in the nocturnal solitary species, and suggests that males are more sensitive to context of stimulation than females. 


\section{Exploring the behavioral reactions to a mirror in the}

2 nocturnal grey mouse lemur: sex differences in

\section{3 avoidance}

4 *Pauline B. Zablocki-Thomas ${ }^{1,2}$ (OrcID: 0000-0002-2372-4760), *Grégoire Boulinguez-

5 Ambroise $^{1}$ (0000-0002-9855-2887), Justine Mézier ${ }^{1}$, Camille Pacou ${ }^{1}$, Anthony Herrel ${ }^{1,3}$ (0000-

6 0003-0991-4434), Fabienne Aujard ${ }^{1}$ (0000-0003-1074-7784), Emmanuelle Pouydebat ${ }^{1}$ (0000-

7 0002-0542-975X)

8 'UMR 7179, Département d'Écologie et de Gestion de la Biodiversité, Museum national

9 d'Histoire naturelle, PARIS, France

$10{ }^{2}$ California National Primate Research Center, Davis, CA, United States

$11{ }^{3}$ Evolutionary Morphology of Vertebrates, Ghent University, K.L. Ledeganckstraat 35, B-9000

12 Ghent, Belgium.

$13 *$ First authors that contributed equally to the study.

14 Corresponding Author:

15 Grégoire Boulinguez-Ambroise

16 Email address: gregoire.boulinguez-ambroise@,cri-paris.org 


\section{Abstract}

Most mirror-image stimulation studies (MIS) have been conducted on social and diurnal animals in order to explore self-recognition, social responses, and personality traits. Small, nocturnal mammals are difficult to study in the wild and are under-represented in experimental behavioral studies. In this pilot study, we explored the behavioral reaction of a small nocturnal solitary forager - the grey mouse lemur (Microcebus murinus) - an emergent animal model in captivity. We assessed whether MIS can be used to detect a repeatable behavioral reaction, whether individuals will present a similar reaction toward a conspecific and the mirror, and whether males and females respond similarly. We tested 12 individuals (6 males and 6 females) twice in three different contexts: with a mirror, with a live conspecific, and with a white board as a neutral control. We detected significant repeatability for the activity component of the behavioral reaction. There was a significant effect of the context and the interaction between presentation context and sex for avoidance during the first session for males but not for females. Males avoided the mirror more than they avoided a live conspecific. This pilot study opens a discussion on the behavioral differences between males and females regarding social interactions and reproduction in the nocturnal solitary species, and suggests that males are more sensitive to context of stimulation than females.

\section{Introduction}

Mirror-image stimulation (MIS) and mirror tests are used to study multiple questions in the study of animal behavior. The most common and well-known use is to determine whether animals are capable of self-recognition (Gallup, 1968), i.e., whether they display a self-oriented response to a mark made on a body part that is only visible in a mirror (i.e., mark test). Such selfengaged response has been tested in a large range of species over the past decades, including Asian elephants (Elephas maximus), marmots (Marmota flaviventris), chimpanzees (Pan troglodytes), corvids (Pica pica), dolphins (Tursiops truncatus), and ants (Formicidae) (Plotnik et al., 2006, Svendsen \& Armitage, 1973, Gallup, 1970, Prior et al., 2008, Reiss \& Marino, 2001, Cammaerts Tricot \& Cammaerts, 2015). The results brought out that many species are presenting social behaviors as a response to the mirror presentation, including aggressive behaviors (e.g., tooth chatter and lunges in marmots; jaw-clapping and charging in dolphins). Yet, only a handful of 
46 species appears capable of self-recognition, specifically apes species (Gallup et al., 2002), killer 47 (Orcinus orca) and false killer (Pseudorca crassidens) whales (Delfour \& Marten, 2001), and

Individuals can be more or less attracted by the image of a conspecific, which depends on how they look and how they behave (Armitage, 1986; Réale et al., 2007; Cattelan et al., 2017). Using a mirror instead of a conspecific has the advantage of standardizing the reaction of the conspecific image, while removing most acoustic and olfactory cues (Svendsen \& Armitage, 1973). For instance, in titi monkeys (Plecturocebus cupreus), a study showed that using MIS successfully simulated the presence of conspecifics, suggesting that the use of MIS could be also useful in other primate species (Fisher-Phelps et al., 2016). Mirror tests are relevant experiments to investigate personality traits as well (Réale et al., 2007; Wilson et al., 2011). Specifically, mirror tests are used to describe the aggression and sociability axis, and have been extensively used in marmots (Marmota flaviventer) and squirrels (Sciurus carolinensis, Sciurus vulgaris) (Svendsen \& Armitage, 1973; Armitage, 1986, Wauters et al., 2019, Petelle et al., 2019; Santicchia et al., 2020). Research on animal personality aims to understand why some individuals respond consistently in a different manner compared to others in a given context. Personality traits can be quantified for each individual by carrying out specific tests. For example, the largely used open field tests and novel-object tests provide data on exploration behavior, activity or boldness (Dammhahn, 2012; Dammhahn \& Almeling, 2012; Highcock \& Carter, 2014; Perals et al., 2017; Savidge \& Bales, 2020), but other experiments like handling (Verdolin \& Harper, 2013) and tonic immobility (Erhard \& Mendl, 1999) are used to quantify aggressiveness in various taxa. Aggression and sociability, as personality traits, have also been addressed using mirror tests; for example, in yellow-bellied marmots (Marmota flaviventris; Svendsen \& Armitage 1973; Armitage, 1986) or in rainbow kribs (Pelvicachromis pulcher; Scherer et al., 2016), making MIS a valuable tool in this area of investigation as well.

Although a wide range of taxa have been tested using MIS (i.e., facing a full-sized mirror in their environment, either in the wild or captivity), predominantly diurnal and social species have 
76 been tested to date. These include fish (guppies, Poecilia reticulata; Cattelan et al., 2017; Siamese 77 fighting fish, Betta splendens; Takeuchi et al. 2010), molluscs (Sepia officinalis; Palmer et al., 78 2006), birds (magpies, Pica pica; Prior et al., 2008), insects (ants, Hymenoptera, Formicidae; 79 Cammaerts Tricot \& Cammaerts, 2015), as well as numerous mammals (Asian elephants; Plotnik 80 et al., 2006; marmots; Svendsen \& Armitage, 1973; great apes; Gallup, 1970; Westergaard \& 81 Hyatt, 1994; Hanazuka et al., 2018; marine mammals, Tursiops truncatus; Reiss \& Marino, 2001; 82 but also in the solitary giant panda, Ailuropoda melanoleuca; Ma et al., 2015). The only MIS study 83 to date using strepsirrhines focused on a nocturnal species: the Garnett's greater bush baby 84 (Otolemur garnettii; Becker et al., 1999). When close to the mirror, and oriented to it, bush babies 85 showed threat gestures and bipedal postures. Moreover, males were found to engage more in scent 86 marking than females in immediate proximity to the mirror (Becker et al., 1999). Thus, the authors 87 of the study concluded that, even if nocturnal strepsirrhines rely more on olfactory and auditory 88 stimuli for social communication, they also appear to be sensitive to mirror-image simulations 89 (Becker et al., 1999), as compared to diurnal species that rely predominantly on vision for 90 91 2020).

Here, we present data on MIS on the nocturnal grey mouse lemur (Microcebus murinus). This species lives in dry deciduous forests along the western and southern coasts of Madagascar 94 (Mittermeier \& Nash, 2010). Although this small lemuriform (i.e., about $80 \mathrm{~g}$ ) forages alone during 95 the night, it is not solitary and shows a complex and yet poorly understood social structure 96 (Radespiel 2000; Schwab 2000; Weidt et al. 2004; Dammhahn \& Kappeler 2005). While mouse 97 lemurs rely mainly on auditory and olfactory cues for social communication, their visual system 98 is well developed and used when foraging in the complex, discontinuous 3D-arboreal environment 99 (Pariente, 1979; Schilling, 2000; Ho et al., 2020). Visual cues are also used for social 100 communication (Peter et al., 1977; Shilling, 2000); for instance: tail-lashing (moving tail from side 101 to side) and mouth opening are used to threaten, folded ears and tail-curling are used to express 102 fear, and genitalia presentation is used in a sexual context (female sexual behavior). During the 103 day, M. murinus shows gregariousness in tree holes. Sleeping groups constitute the basal social 104 unit and can contain up to 16 individuals (Radespiel et al. 2001; Weidt et al. 2004). It has been 105 reported that sleeping groups are stable, as well as home ranges, suggesting stable social 
106 relationships and individual recognition (Radespiel 2000; Weidt et al. 2004). Female sleeping 107 groups are usually made up of closely related individuals, showing a matrilineal social structure 108 (Radespiel et al. 2001). Indeed, sleeping groups seem to be mostly made of individuals of the same

109

110

111

112

113

114

115

116

117

118

119

120

121

122

123

124

125

126

127

128

129

130

131

132

133 sex, but this changes during the mating season (Martin, 1973). Mouse lemurs have one breeding season a year and females can have more than one estrus cycle per season (Blanco, 2008; Wrogemann et al. 2001). Like most lemuriforms, females are dominant over males (Kappeler, 1993) and present an increased aggressiveness towards the male solicitations before becoming receptive (Gomez et al., 2012). Despite these elements, the social life of this nocturnal species remains poorly understood. A better understanding of its social behavior would be of interest and might provide insights into the evolution of sex differences, but also, it may further help improve breeding and maintenance of the species in captivity (Languille et al., 2012; Roberts, 2019).

In this study, we aimed to compare the response of $M$. murinus to a MIS and to a live conspecific, as well as assessing possible differences between sexes in their social response. We quantified several behaviors related to activity, approach, and vigilance. We tested whether behaviors were consistent across time and contexts within individuals, and tested for possible sexrelated differences in behavior. We predicted that (1) this experiment will allow for the detection of repeatable reactions across time and context within individuals, (2) individuals will present a similar reaction toward a conspecific and the mirror, (3) males and females will differ their responses in the mirror context (Mir). The results of this pilot study will allow us to assess whether MIS is a relevant tool to investigate social behavior in M. murinus.

\section{Materials \& Methods}

\section{Subjects}

We studied twelves (six males and six females) captive grey mouse lemurs (Microcebus murinus) that were born and raised in the colony of the UMR 7179 (CNRS/MNHN) of the Museum National d'Histoire Naturelle (Brunoy, France, Agreement F91-114-1). We conducted the study in July and August 2018. Individuals lived in groups of two to seven individuals in large aviaries (167 x $60 \mathrm{x}$ $70 \mathrm{~cm}$ ), maintained under artificial light conditions, allowing control over season and the period of day (photoperiod). Besides, the aviaries were enriched with fresh leafy branches and wooden 
134 nest boxes, while their temperature was maintained around $25^{\circ} \mathrm{C}$ and the humidity around $30 \%$. 135 Food and water were available ad libitum (including fresh fruits, a milky mixture, and 136 mealworms). The behavioral tests took place after the reproductive season, so that females were 137 not in estrus. Animals were individually identified via ear tags.

\section{Behavioral tests}

139 We built two behavioral test boxes made out of Plexiglas (40 x $25 \times 25 \mathrm{~cm})$, each with one lateral 140 panel and a transparent front panel. A mirror $(20 \times 20 \mathrm{~cm})$ or an opaque board could be placed in 141 front of the transparent panel (Figure 1). The boxes were divided into three equally sized zones,

142 separated by an imaginary line: Zone 1 with the transparent panel/mirror/opaque board, Zone 2 in 143 the middle, and Zone 3 at the opposite side of the box. The size of the mirror allowed the individual 144 to see its whole body.

145 After removing the subject from its individual nest box, we opened the test box and placed the 146 subject in Zone 3. The trial started when closing the test box. The behavioral experiment lasted 30 147 minutes and consisted of letting the subject move freely in the test box in three contexts: (1) with 148 a mirror (Mir); (2) with lateral panel opaque as a neutral control context (OF); and (3) a live control 149 context (T), where another individual of the same sex is visible through the transparent Plexiglas. 150 For the $\mathrm{T}$ context, a live conspecific from the same age and sex class was in an identical box 151 adjacent to the subject's box. Individuals that were tested together were not familiar to each other, 152 meaning that they did not live in the same aviary. This context (i.e., conspecific) allowed us to 153 collect data on two animals at the same time to maximize data collection. We selected a sample including pairs of individuals of same sex, age and corpulence for this purpose. We recorded behavioral tests using a digital camera (SONY Handycam DCR-SR75) with the infrared (IR) mode activated, as we filmed in the dark. We added an IR spotlight to gain visibility. We proceeded to a blind analysis (meaning that the scorer did not know the identity or sex of the individual) to quantify behaviors using the software VLC media player.

During the three contexts, we recorded eight behavioral variables related to activity, approach, and vigilance (Table 1). Individuals were tested twice in each context for a total of six tests per individual, in random order (the three contexts for the first session, and after that the three contexts 
162 for the second session were randomized each time). We conducted repetitions within an interval 163 of 10 to 22 days. However, two females did not participate in all six tests because of health issues: 164 one female experienced Mir and T only once, and another female was run in T only once. We kept 165 these records as NA for the analysis.

\section{Statistical analysis}

167 We first conducted a principal component analysis (PCA) on the eight variables and subsequently 168 conducted a VARIMAX rotation on the recorded behaviors to reduce the number of behavioral variables to a smaller number of independent variables (Buadev et al., 2010). The first three principal components - named PC1, PC2 and PC3- accounted for 47.3\%, 13.4\% and 27.0\% of the behavioral variance, respectively. We kept only three components as jointly they explained more than $80 \%$ of the total variability. We used the acp.varimax() function provided by "psych" package (Revelle, 2019) with R software version 3.6.0 (Rstudio Version 1.1.456).

174 Subsequently, we estimated principal component repeatability as an intraclass correlation coefficient, which is the ratio of the interindividual variance to the sum of inter- and intraindividual variances (Nakagawa \& Schielzeth, 2010). For that purpose, we ran a linear mixed effects model, with only individual as random effect and no fixed effect. We used the rpt() function in the "rptR package" (Nakagawa \& Schielzeth, 2010).

179 We used a non-parametric two-way ANOVA with context as a repeated factor and sex as an independent factor. We used the package nparLD and the f1.ld.f1 function (Noguchi et al. 2012). When the ANOVA was significant, we conducted a Friedman test to detect between which test contexts a difference was observed (for both sexes, and for females and males separately) followed by a post-hoc correction with the pgirmess package (Giraudoux et al., 2018) and the friedmanmc function (proceeding to pairwise comparison followed by the observed difference between the rank sums (Sidney, 1957)). We used $\alpha=0.05$ as the threshold for statistical significance. Boxplots were drawn in R using the geom_boxplot() function provided by ggplot2 package (Wickham, 2016). 
All subjects included in the study were born and reared in captivity in the colony of the UMR 7179 (CNRS/MNHN) of the Muséum National d'Histoire Naturelle (MNHN, Brunoy, France, Agreement F91-114-1). Observations and animal handling were performed in accordance with the relevant MNHN guidelines and the European Union regulations (Directive 2010/63/EU).

192

193

194

195

196

197

198

199

200

201

202

203

204

205

206

207

208

209

210

211

212

213

214

\section{Results}

The first principal component (PC1) loaded positively with activity-related behaviors: high values of PC1 correspond to a higher number of moves, a greater occurrence and duration of rearing, a higher occurrence of approach and mirror contacts. High values of PC3 correspond to a longer latency to start exploration and to approach the mirror. By contrast, the second component (PC2) was highly positively determined by time spent in the mirror zone (Zone 1), meaning that individuals with a low PC2 value avoided Zone 1 more than individuals with high PC2 scores (Table 2). PC1 can be interpreted as an "activity" score, PC2 as an "approach" score, and PC3 as an "anxiety" score. Next, we tested for repeatability across the three contexts and sessions (two sessions for three tests contexts) using the principal components scores for each individual. Only PC1 was repeatable (Table 3).

As PC1 was repeatable, we averaged it over the two sessions by individual to have a more representative and unique estimator of activity. For PC2 and PC3, we kept only data from each subject's first session. The repeated measures ANOVA on the first principal component score for each individual $(\mathrm{PC} 1)$ revealed no effect of sex $(\mathrm{P}>0.40, \mathrm{df}=1, \mathrm{~F}=0.69)$ or context $(\mathrm{P}>0.92$, $\mathrm{df}=1.9, \mathrm{~F}=0.07)$, and no interaction between the two factors $(\mathrm{P}>0.30, \mathrm{df}=1.9, \mathrm{~F}=1.21)$. For $\mathrm{PC} 3$ (Session 1), there was also no effect of $\operatorname{sex}(\mathrm{P}>0.98, \mathrm{df}=1, \mathrm{~F}=0.0008)$ or context $(\mathrm{P}>0.96$, $\mathrm{df}=1.9, \mathrm{~F}=0.038)$ and no interaction between the two factors $(\mathrm{P}>0.82, \mathrm{df}=1.9, \mathrm{~F}=0.18)$. For

PC2 (Session 1), although we found no effect of sex $(P>0.49, \mathrm{df}=1, \mathrm{~F}=0.49)$, we found a significant effect of context $(\mathrm{P}=0.009, \mathrm{df}=1.3, \mathrm{~F}=5.79)$, with a higher approach score in the $\mathrm{T}$ context (with a conspecific) and a lower approach score in the Mir (mirror) context (Figure 2). In addition, an interaction between the two factors $(\mathrm{P}=0.03 \mathrm{df}=1.3, \mathrm{~F}=4.01)$ was detected, with males having a higher approach score (higher PC2) in the T context compared to the Mir context, 
215 whereas females spent less time in Zone 1 in Mir and T contexts compared to the OF context 216 (Figure 2).

217

218

219

220

221

222

223

224

225

226

227

228

229

230

231

232

233

234

235

236

237

238

239

240

241

242

Finally, using a Friedman test followed by a post-hoc correction on PC2 for males and females together, and for males only, we found a significant effect of the test context, with individuals spending more time in Zone 1 during the context with the conspecific than with the mirror (all individuals: Friedman chi-squared $=7,09$, $\mathrm{df}=2, \mathrm{P}<0.029$; males: Friedman chisquared $=10, \mathrm{df}=2, \mathrm{P}<0.0067)$. There was no difference between the three contexts for females only (Friedman chi-squared $=1.33, \mathrm{df}=2, \mathrm{P}=0.51)$.

\section{Discussion}

Our results showed a significant repeatability for "activity-related behaviors". Indeed PC1, that mainly showed strong loadings for body movements and displacements, was repeatable across time and contexts, and could thus be considered as related to overall "activity". PC2 was mostly determined by the time spent in Zone 1 (i.e., close to the mirror/conspecific) and was interpreted as an "approach score", while PC3 was mostly determined by the latency to start moving and can be described as an "anxiety score". Both PC2 and PC3 were not significantly repeatable. In males, but not in females, we detected a differential approach score between the mirror and the live conspecific. Only one case of an attack toward a mirror was reported in a female during a preliminary test (personal observation).

We predicted repeatable reactions across time and contexts within individuals. In fact, we found the first component (i.e., PC1, "activity score") to be repeatable, being in line with previous studies on captive and wild M. murinus that have reported significant but moderate repeatability for behavioral traits: 0.33 for emergence latency, 0.28 for agitation, 0.25 for latency to start exploration in an open field test (Thomas et al., 2016; Zablocki-Thomas et al., 2018). Consequently, we suggest that MIS could be a useful tool in understanding personality in this species. Conducting other behavioral tests would be necessary to be able to integrate our "activity score" to the animal personality framework. However, this personality axis related to activity appears to be relatively easily detectable in M. murinus (Dammhahn, 2012). Sociability is less easy to quantify in this solitary forager since it is active during the night and thus more difficult to 
243 observe than diurnal species. We used MIS to attempt to detect within-individual repeatable 244 behaviors linked with sociability across time in this species as it has previously been done in other 245 mammal species (Svendsen \& Armitage, 1973; Armitage, 1986, Wauters et al., 2019, Petelle et 246 al., 2019; Santicchia et al., 2020). However, we did not detect repeatable social behaviors such as 247 approaching the mirror/conspecific or scent marking as documented for bush babies (Becker et al., 248 1999).

249 Our second prediction was that the individuals would present a similar reaction to the mirror and 250 to the conspecific. We compared reactions to a mirror with those to a live conspecific, which could 251 either elicit a similar reaction because of the visual similarity with the image, or a different reaction 252 because of the lack of auditory or olfactory cues (Svendsen \& Armitage, 1973). In our study, we 253 found no significant difference in the composite behaviors PC1 (i.e., activity) and PC3 (i.e., 254 anxiety) of M. murinus when presenting either a mirror, a conspecific, or an opaque wall. However, 255 there was a significant effect of context and a significant interaction between sex and context for 256 PC2 (i.e., the approach score): (1) overall, compared to the opaque wall, individuals approached 257 the conspecific and avoided the mirror, and (2) males responded differently to the conspecific 258 compared to the mirror, showing more approaches to the former. We did not expect to find such 259 differences between the mirror context and the positive control context (i.e., with conspecific) in 260 males, since mirror tests are supposed to simulate the presence of a conspecific (Svendsen \& 261 Armitage, 1973). Our finding is not consistent with a study on West African cichlids, which posits 262 that mirror and conspecific presentation should elicit comparable responses (Scherer et al., 2016). 263 However, Cattelan et al. (2017) tested the accuracy of the comparison of mirror tests to tests with 264 living conspecifics in guppies (Poecilia reticulata). They measured sociability in both contexts, 265 and showed that the MIS may be less reliable than tests with conspecifics if some improvements 266 were not made to simulate more naturalistic situations (Cattelan et al., 2017). Other studies have 267 correlated the aggressive response to a mirror with the aggressive response in tests with a 268 conspecific (e.g., Scherer et al., 2016). However, animals tended to be more aggressive toward the 269 conspecific than to mirror images. Altogether, these studies seem to show that the behavioral 270 response toward a mirror compared to other social/control stimulation is species-specific and 271 presumably dependent on the ecology of the animal (e.g., social system, territoriality, 272 communication, habitat use). The fact that nocturnal mammals rely mostly on olfactory and 
273 auditory cues for social communication does not appear to explain the difference in the reaction 274 across contexts. Other nocturnal animals tended to show differences to social video stimulations 275 versus non-social stimulations. For example, rats spent more time in the presence of a mirror or a 276 video image than in an empty chamber without image stimulation, showing that these animals can 277 visually discriminate between the absence and the simulated presence of a conspecific, and 278 respond as if they were in presence of a conspecific (Yakura et al., 2018).

279 Our unexpected results on the reaction toward a conspecific and a mirror in $M$. murinus suggest 280 that the MIS should be validated and studied extensively for each species, as was previously 281 suggested for fish (Balzarini et al., 2014). Other studies using MIS conducted with non-primate 282 mammals (marmots (Marmota flaviventer) and squirrels (Sciurus carolinensis, Sciurus vulgaris) 283 (Svendsen \& Armitage, 1973; Armitage, 1986, Wauters et al., 2019, Petelle et al., 2019; Santicchia 284 et al., 2020)) use this approach as a routine tool to assess personality. For example, in Wauters et 285 al. (2019), the authors use MIS to show that personality in squirrels is impacted by ecological 286 competition between species. In primates, the majority of the studies focus on the theory of mind 287 (De Veer et al. 1999) and do not attempt to demonstrate that MIS can be used to simulate the 288 presence of a conspecific. In captive titi monkeys (Plecturocebus cupreus), where the absence of 289 self-recognition has been acknowledged (Fisher-Phelps et al., 2016), MIS represents a valuable 290 tool too to study social relationships. In this species (Mercier et al., 2020), mirror presentation 291 elicits reactions similar to the presentation of a couple of conspecific strangers (e.g., coordinated 292 tail-lashing within pair-mates).

293 Interestingly, we found that males responded differently to the conspecific compared to the mirror, 294 while female behaviors did not change significantly across contexts, confirming our third 295 prediction. Our results are not fully consistent with a report on male bush babies (Becker et al., 296 1999) approaching the mirror more than females. While we also found a difference between sexes, 297 our results suggest that males distinguished between the mirror and the live conspecific, whereas 298 females did not, with males avoiding the mirror. We cannot fully compare our study with the study 299 on bush babies since they did not use a context with a living conspecific. Here, we can only 300 extrapolate our result on captive to wild M. murinus. From an ecological perspective, a difference 301 between male and female M. murinus in the reaction to the mirror and to a conspecific could be 302 interpreted as a difference in behavioral strategy between the sexes (Eberle and Kappeler, 2004a, 
303 b). Females, for example, sleep in larger groups with related individuals whereas males are more 304 solitary (Radespiel et al. 2001). In this species, social interactions between sexes during the active 305 phase are rare and typically occur only once a year during the reproductive season (Perret, 1997; 306 Andrès et al., 2003), when the female is sexually receptive for approximately 24 hours. Adult male 307 M. murinus (over 3 years of age) are bolder than females in the wild, which could be explained by 308 a higher propensity to take risks when looking for a mate (Dammhahn, 2012). Besides, avoidance 309 is a submissive behavior and female are dominant over males (Microcebus sp.), so that spatial 310 conflicts are most of the time won by females (Radespiel \& Zimmermann, 2001). This may explain 311 why females present less difference between the three contexts in our experiment. Further 312 investigations during the reproductive season would be relevant, as during this period, individuals 313 are actively looking for mates and are more sensitive to social stimulation. Alternatively, male $M$. 314 murinus avoiding the zone close to the mirror could suggest their incapacity to interpret the 315 simulated presence of a conspecific in a mirror.

316 We know of no other published MIS experiments to study behavioral reaction differences between 317 the sexes, but several studies involving open field tests, or novel object tests have been conducted 318 and linked to variation in age, sex, and morphology in wild and captive M. murinus (Dammhahn, 319 2012; Dammhahn \& Almeling, 2012; Thomas et al., 2016). In the wild (Dammhahn, 2012) young 320 (<3 years) male M. murinus are bolder than older males and females since they approach novel 321 objects faster and for longer, showing an effect of age and sex on M. murinus reactions. In captivity 322 lighter and smaller individuals were the ones that started exploring new environments showing an 323 effect of morphology on exploration behavior (Thomas et al., 2016). In the present study, we could 324 not investigate the effect of age considering the limited sample size of our study. The large 325 variation of behaviors found in our data could also be explained by this limited sample size.

326 Besides, we did not conduct other biological measures than behavioral measurement. For future 327 research, physiological measurements such as stress-levels (e.g., cortisol levels) may be relevant 328 to better describe the sexual dimorphism in the reaction toward the mirror. In addition, as brain 329 imaging studies are now possible and more easily accessible with animals (Ferris, 2014; Maninger 330 et al., 2017; Cook et al., 2018), we may consider using PET scans to further assess whether males 331 and females are interpreting the situation differently (i.e., determining brain areas involved in the 332 different responses). 
333 Conclusions

334 This pilot study provides first data on the relevance of using MIS to study social behaviors in the 335 nocturnal grey mouse lemur (Microcebus murinus), for which data on sociality are particularly 336 lacking. Here, we explored whether M. murinus responded with the same reaction toward a mirror 337 presentation and in the presence of a conspecific, and assessed potential sex differences. Whereas 338 females responded in the same manner in all contexts, males did not, avoiding the mirror more 339 than a live conspecific, suggesting a contrast in their social interactions. Such findings offer an 340 insight on sex differences in social behaviors in this emergent model, that may help for both 341 designing future research protocols and managing colonies in captivity.

\section{Acknowledgements}

343 We would like to thank Sandrine Gondor-Bazin, Eric Guéton, and Lauriane Dezaire for helping 344 with the daily care of the animals and Isabelle Hardy for the management of the colony database. 345 We warmly thank Hughes Clamouze for helping us build the experimental device. We also thank 346 the ENS of Lyon, Karen Bales' lab (UC Davis, CNPRC) and the Fyssen Foundation for their 347 financial support to PZT.

\section{References}

349 Andrès M, Solignac M, Perret M. 2003. Mating System in Mouse Lemurs: Theories and Facts, 350 Using Analysis of Paternity. Folia Primatologica 14:355-366. DOI: 10.1159/000073319.

Armitage K. 1986. Individuality, Social Behavior, and Reproductive Success in Yellow-Bellied Marmots. Ecology 67:1186-1193. DOI: 10.2307/1938674.

Balzarini, V., Taborsky, M., Wanner, S., Koch, F., Frommen, J.G. 2014. Mirror, mirror on the wall: the predictive value of mirror tests for measuring aggression in fish. Behav Ecol Sociobiol 68, 871-878. https://doi.org/10.1007/s00265-014-1698-7

Becker, L., Watson, S.L., Ward, J.P. 1999. Mirror-Directed Social Behaviors of Garnett's Greater Bush Baby (Otolemur garnettii). International Journal of Primatology 20:633-650. DOI: 10.1023/A:1020744500711. 
359 360

361 362 363 364 365 366 367 368 369 370

Blanco, M.B. 2008. Reproductive schedules of female Microcebus rufus at Ranomafana National Park, Madagascar. Int J Primatol 29(2):323-38.

Brandl JL. 2018. The puzzle of mirror self-recognition. Phenomenology and the Cognitive Sciences 17:279-304. DOI: 10.1007/s11097-016-9486-7.

Budaev, S.V. 2010. Using Principal Components and Factor Analysis in Animal Behaviour Research: Caveats and Guidelines. Ethology, 116: 472-480.

Cammaerts Tricot, M.-C., and R. Cammaerts. 2015. Are ants (Hymenoptera, Formicidae) capable of self-recognition? Journal of Science 5:521-532.

Cattelan S, Lucon-Xiccato T, Pilastro A, Griggio M. 2017. Is the mirror test a valid measure of fish sociability? Animal Behaviour 127:109-116. DOI: 10.1016/j.anbehav.2017.03.009.

Cook P, Prichard A, Spivak M, Berns G.S. 2018. Jealousy in dogs? Evidence from brain imaging. Animal Sentience 22, 1-14.

Dammhahn M., Kappeler P.M. 2005. Social system of Microcebus berthae, the world's smallest primate. Int J Primatol 26(2):407-35.

Dammhahn M. 2012. Are personality differences in a small iteroparous mammal maintained by a life-history trade-off? Proceedings. Biological sciences / The Royal Society 279:2645-51. DOI: $10.1098 /$ rspb.2012.0212.

Dammhahn, M., \& Almeling, L. 2012. Is risk taking during foraging a personality trait? A field test for cross-context consistency in boldness. Animal Behaviour, 84(5), 1131-1139.2012.

delBarco-Trillo, J., Drea, C.M. 2014. Socioecological and phylogenetic patterns in the chemical signals of strepsirrhine primates. Animal Behaviour 97: 249-253.

Delfour F, Marten K. 2001. Mirror image processing in three marine mammal species: Killer whales (Orcinus orca), false killer whales (Pseudorca crassidens) and California sea lions (Zalophus californianus). Behavioural Processes 53,181-190. DOI: 10.1016/S0376- 
384

385

386

387

388

389

390

391

392

393

394

395

396

397

398

399

400

401

402

403

404

405

406

407

De Veer, M. W., \& Van den Bos, R. 1999. A critical review of methodology and interpretation of mirror self-recognition research in nonhuman primates. Animal Behaviour, 58(3), 459468.

Doyle G.A., Martin, R.D. 1979. The Study of Prosimian Behavior. Academic Inc. Press.

Drea, C.M. 2020. Design, delivery and perception of condition-dependent chemical signals in strepsirrhine primates: implications for human olfactory communication. Philosophical Transactions of the Royal Society B 375.1800: 20190264.

Eberle, M., \& Kappeler, P. M. 2004a. Sex in the dark: determinants and consequences of mixed male mating tactics in Microcebus murinus, a small solitary nocturnal primate. Behavioral Ecology and Sociobiology, 57(1), 77-90.

Eberle, M., \& Kappeler, P. M. 2004b. Selected polyandry: female choice and inter-sexual conflict in a small nocturnal solitary primate (Microcebus murinus). Behavioral Ecology and Sociobiology, 57(1), 91-100.

Erhard, H.W., Mendl, M. 1999. Tonic immobility and emergence time in pigs: More evidence for behavioural strategies. Applied Animal Behaviour Science 61, 227-237. DOI: 10.1016/S0168-1591(98)00196-8.

Ferris CF. 2014. Using Awake Animal Imaging to Understand Neural Circuits of Emotion: Studies Ranging from Maternal Care to Aggression. In: New Frontiers in Social Neuroscience. Research and Perspectives in Neurosciences. Springer, Cham, 600-601. DOI: https://doi.org/10.1007/978-3-319-02904-7 7.

Fisher-Phelps, M.L., Mendoza, S.P., Serna, S., Griffin, L.L., Schaefer, T.J., Jarcho, M.R., Ragen, B.J., Goetze. L.R., and Bales, K.L. 2016. Laboratory simulations of mate-guarding as a component of the pair-bond in male titi monkeys, Callicebus cupreus. American journal of primatology, 78(5), 573-582. 
408 Gallup GG. 1968. Mirror-image stimulation. Psychological bulletin 70:782-793. DOI:

$409 \quad 10.1037 / \mathrm{h} 0026777$.

410 Gallup, G. G. 1970. Chimpanzees: self-recognition. Science 167:86-87.

411 Gallup GG, Anderson JR. 2018. The "olfactory mirror" and other recent attempts to demonstrate 412 self-recognition in non-primate species. Behavioural Processes 148:16-19. DOI:

$413 \quad$ 10.1016/j.beproc.2017.12.010.

414 Gallup GG, Anderson JR, Shillito DJ. 2002. The Mirror Test. The cognitive animal: Empirical 415 and theoretical perspectives on animal cognition:325-33. DOI:

$416 \quad 10.1017 /$ CBO9781107415324.004.

417 Giraudoux P, Antonietti J-P, Beale C, Pleydell D, Treglia M. 2018. pgirmess: Spatial Analysis 418 and Data Mining for Field Ecologists.

419 Gomez D, Huchard E, Henry P-Y, Perret M. 2012. Mutual mate choice in a female-dominant and 420 sexually monomorphic primate. American journal of physical anthropology 147:370-9.

421 DOI: 10.1002/ajpa.21653.

422

423

424

425

426

427

428

429

430

431

Hanazuka, Y., M. Shimizu, H. Takaoka, and A. Midorikawa. 2018. Orangutans (Pongo pygmaeus) recognize their own past actions. Royal Society Open Science 5:0-6.

Heyes CM. 1994. Reflections on self-recognition. Animal Behaviour 47:909-919.

Highcock L, Carter AJ. 2014. Intraindividual Variability of Boldness Is Repeatable across Contexts in a Wild Lizard. PloS one 9:e95179. DOI: 10.1371/journal.pone.0095179.

Ho, C.L.A., Zimmermann, R., Florez Weidinger, J.D., Prsa, M., Schottdorf, M., Merlin, S., Okamoto, T., Ikezoe, K., Pifferi, F., Aujard, F., Angelucci, A., Wolf, F. \& Huber, D. 2020. Orientation Preference Maps in Microcebus murinus Reveal Size-Invariant Design Principles in Primate Visual Cortex. Current Biology https://doi.org/10.1016/j.cub.2020.11.027 
432 Kappeler PM. 1993. Female dominance in primates and other mammals. Perspectives in 433 ethology 10:143-158.

434 Languille, S., Blanc, S., Blin, O., Canale, C. I., Dal-Pan, A., Devau, G., ... \& Aujard, F. (2012). 435 The grey mouse lemur: a non-human primate model for ageing studies. Ageing research 436 reviews, 11(1), 150-162.

437 438

439

440

441

442

443

444

445

446

447

448

449

450

451

452

453

454 455

456

Ma X, Jin Y, Luo B, Zhang G, Wei R, Liu D. 2015. Giant pandas failed to show mirror selfrecognition. Animal Cognition:713-721. DOI: 10.1007/s10071-015-0838-4.

Maninger N, Mendoza S, Williams D, Mason W, Cherry S, Rowland D, Schaefer T, Bales KL. 2017. Imaging, Behavior and Endocrine Analysis of "Jealousy" in a Monogamous Primate. Frontiers in Ecology and Evolution 5:119. DOI: 10.3389/fevo.2017.00119.

Martin, R.D. 1973, A review of the behaviour and ecology of the lesser mouse lemur (Microcebus murinus, J. F. Miller 1777). In R. P. Michael \& J. H. Crook (Eds.), Comparative ecology and behaviour of primates (pp. 1-68). London: Academic Press.

Mercier, F., Witczak, L. R., \& Bales, K. L. 2020. Coppery titi monkey (Plecturocebus cupreus) pairs display coordinated behaviors in response to a simulated intruder. American Journal of Primatology, e23141.

Mittermeier R.A., Nash S.D. 2010. Lemurs of Madagascar. Conservation International.

Nakagawa S, Schielzeth, H. 2010. Repeatability for Gaussian and non-Gaussian data: a practical guide for biologists. Biological Reviews 85: 935-956

Noguchi K, Latif M, Thangavelu K, Konietschke F, Gel YR, Brunner E. 2012. nparLD: Nonparametric Analysis of Longitudinal Data in Factorial Experiments. J Stat Softw. 2012;50(12):1-23. DOI: 10.18637/jss.v050.i12

Palmer, M.E., Calvé, M.R., \& Adamo, S.A. 2006. Response of female cuttlefish Sepia officinalis (Cephalopoda) to mirrors and conspecifics: Evidence for signaling in female cuttlefish. Animal Cognition, 9(2), 151-155. 
457 Pariente, G.F. 1979. The role of vision in prosimian behaviour. In G. A. Doyle \& R. D. Martin 458 (Eds.), The study of prosimian behavior (pp. 411-469). New York: Academic Press.

459 Perals D, Griffin AS, Bartomeus I, Sol D. 2017. Revisiting the open-field test: what does it really 460 tell us about animal personality? Animal Behaviour 123:69-79. DOI:

$461 \quad$ 10.1016/j.anbehav.2016.10.006.

462 Perret M. 1997. Change in photoperiodic cycle affects life span in a prosimian primate 463 (Microcebus murinus). Journal of biological rhythms 12:136-145. DOI:

$464 \quad 10.1177 / 074873049701200205$.

465

466

467 468

469

470

471

472

473

474

475

476

477

478

479

480

481

Petelle, M. B., Martin, J. G., \& Blumstein, D. T. 2019. Mixed support for state maintaining risky personality traits in yellow-bellied marmots. Animal Behaviour, 150, 177-188.

Petter, J.-J., Albignac, R., \& Rumpler, Y. 1977. Mammifères lémuriens (primates prosimiens). In Faune de Madagascar, vol. 44 (pp. 514). Tannarive et Paris: ORSTOM/CNRS

Plotnik JM, de Waal FBM, Reiss D. 2006. Self-recognition in an Asian elephant. Proceedings of the National Academy of Sciences of the United States of America 103:17053-17057. DOI: 10.1073/pnas.0608062103.

Prior H, Schwarz A, Güntürkun O. 2008. Mirror-Induced Behavior in the Magpie (Pica pica): Evidence of Self-Recognition. Plos One 9:1-8. DOI: 10.1371/journal.Citation.

Radespiel, U. 2000. Sociality in the gray mouse lemur (Microcebus murinus) in northwestern Madagascar. Am J Primatol 51(1):21-40.

Radespiel, U. \& Zimmermann E. 2001. Female dominance in captive gray mouse lemurs (Microcebus murinus). American journal of primatology 54:181-92. DOI: 10.1002/ajp.1029.

Radespiel, U., Sarikaya, Z., Zimmermann, E., Bruford, M.W. 2001. Sociogenetic structure in a free-living nocturnal primate population: sex-specific differences in the grey mouse lemur (Microcebus murinus). Behav Ecol Sociobiol 50(6):493-502. 
482 Réale D, Reader SM, Sol D, McDougall PT, Dingemanse NJ. 2007. Integrating animal

483

484

485

486

487

488

489

490

491

492

493

494

495

496

497

498

499

500

501

502

503

504

505

506 temperament within ecology and evolution. Biological reviews of the Cambridge Philosophical Society 82:291-318. DOI: 10.1111/j.1469-185X.2007.00010.x.

Reiss, D., and L. Marino. 2001. Mirror self-recognition in the bottlenose dolphin: a case of cognitive convergence. Proceedings of the National Academy of Sciences of the United States of America 98:5937-42.

Revelle W. 2019. Package "psych" - Procedures for Psychological, Psychometric and Personality Research. $R$ Package version 1.9.12,:1-358.

Roberts, L. 2019. Make way for the mouse lemur. Nature 570, 151-154. https://doi.org/10.1038/d41586-019-01789-0

Santicchia, F., Wauters, L. A., Dantzer, B., Westrick, S. E., Ferrari, N., Romeo, C., Palme, R., Preatoni, D. G., and Martinoli, A. 2020. Relationships between personality traits and the physiological stress response in a wild mammal. Current Zoology, 66(2), 197-204.

Savidge LE, Bales KL. 2020. An Animal Model for Mammalian Attachment: Infant Titi Monkey (Plecturocebus cupreus) Attachment Behavior Is Associated With Their Social Behavior as Adults. Frontiers in Psychology 11. DOI: 10.3389/fpsyg.2020.00025.

Scherer U, Buck M, Schuett W. 2016. Lateralisation in agonistic encounters: do mirror tests reflect aggressive behaviour? A study on a West African cichlid. Journal of Fish Biology 89:1866-1872. DOI: 10.1111/jfb.13069.

Schilling, A. 2000. Organes des sens et communication chez le microcèbe. Primatologie 3:85143.

Schwab, D. 2000. A preliminary study of spatial distribution and mating system of pygmy mouse lemurs (Microcebus cf. myoxinus). Am J Primatol 51(1):41-60.

Sidney S. 1957. Nonparametric statistics for the behavioral sciences. The Journal of Nervous and Mental Disease 125(3).

Peer) reviewing PDF | (2020:10:53606:3:0:NEW 8 Apr 2021) 
507 Siemers BM, Goerlitz HR, Robsomanitrandrasana E, Piep M, Ramanamanjato J-B, Ganzhorn

508 JU, Rakotondravony D, Ramilijaona O. 2007. Sensory Basis of Food Detection in Wild 509 Microcebus murinus. International Journal of Primatology 28:291-304. DOI:

510

511 Svendsen G, Armitage K. 1973. Mirror-image stimulation applied to field behavioral studies. 512 Ecology 54:623-627. DOI: 10.2307/1935349.

513 Takeuchi, Y., Hori, M., Myint, O., \& Kohda, M. (2010). Lateral bias of agonistic responses to 514 mirror images and morphological asymmetry in the Siamese fighting fish (Betta 515 splendens). Behavioural brain research, 208(1), 106-111.

516 Thomas PB, Herrel A, Hardy I, Aujard F, Pouydebat E. 2016. Exploration Behavior and 517 Morphology are Correlated in Captive Gray Mouse Lemurs (Microcebus murinus). 518 International Journal of Primatology 37:405-415. DOI: 10.1007/s10764-016-9908-y.

519 Verdolin JL, Harper J. 2013. Are shy individuals less behaviorally variable? Insights from a 520 521 captive population of mouse lemurs. Primates 54:309-314. DOI: 10.1007/s10329-0130360-8.

522

Wauters, L. A., Mazzamuto, M. V., Santicchia, F., Van Dongen, S., Preatoni, D. G., \& Martinoli,

523

524

525

526

527

528 529

530 531 A. 2019. Interspecific competition affects the expression of personality-traits in natural populations. Scientific reports, 9(1), 1-8.

Weidt A., Hagenah, N., Randrianambinina, B., Radespiel, U., Zimmermann, E. 2004. Social organization of the golden-brown mouse lemur (Microcebus ravelobensis). Am J Phys Anthropol 123(1):40-51.

Westergaard, G. C., and C. W. Hyatt. 1994. The responses of bonobos (Pan paniscus) to their mirror images: Evidence of self-recognition. Human Evolution 9:273-279.

Wickham H. 2016. ggplot2: Elegant Graphics for Data Analysis. Springer-Verlag New York.

Wilson AJ, de Boer M, Arnott G, Grimmer A. 2011. Integrating personality research and animal 
532 contest theory: Aggressiveness in the green swordtail Xiphophorus helleri. PLoS ONE 6.

533 DOI: 10.1371/journal.pone.0028024.

534 Wrogemann D, Radespiel U, Zimmermann E. 2001. Comparison of reproductive characteristics 535 and changes in body weight between captive populations of rufous and gray mouse lemurs. $536 \quad$ Int J Primatol 22(1):91-108.

537 Yakura T, Yokota H, Ohmichi Y, Ohmichi M, Nakano T, Naito M. 2018. Visual recognition of 538 mirror, video-recorded, and still images in rats. PLoS ONE 13:1-11. DOI:

$539 \quad 10.1371 /$ journal.pone.0194215.

540 Zablocki-Thomas PB, Herrel A, Hardy I, Pouydebat LR, Perret M, Aujard F, Pouydebat E. 2018. 541 Personality and performance are affected by age and early life parameters in a small 542 primate. Ecology and Evolution. DOI: 10.1002/ece3.3833. 


\section{LEGENDS:}

544 Figure 1 Behavioral testing box $(40 \times 25 \times 25 \mathrm{~cm})$ used for the mirror-image stimulation study

545 in Microcebus murinus. Three zones were delimited to quantify the behavioral reaction. The 546 sidewall of zone 1 is either transparent facing the other box, a mirror $(20 \times \mathbf{2 0} \mathbf{~ c m})$, or an opaque

547 board, depending on the test context. The animal enters the test area in zone 3.

548 Figure 2 Boxplots of principal component analyses run on behavioral variables quantifying 549 reaction to mirror-image stimulations in female $(n=6)$ and male $(n=6)$ mouse lemurs 550 (Microcebus murinus). The figure shows (A) Principal Component (PC) 1 related to "activity", 551 (B) PC2 which can be described as an "approach score", and (C) PC3 which can be described as 552 an "anxiety score", separated by sex and test context. The contexts were: (Mir) mirror-image 553 stimulation, the mirror visible; (OF) a neutral control with an opaque and lateral panel; (T) a 554 positive control context (i.e., facing a conspecific). Each point represents an individual. The middle 555 line of the boxplot represents the median. The lower and upper hinges correspond to the first and 556 third quartiles (the 25th and 75th percentiles). The upper and lower whisker extends from the hinge 557 to the largest and smallest value no further than $1.5 *$ IQR from the hinge (where IQR is the inter558 quartile range, or distance between the first and third quartiles). $P$-value $*<0.05 ; * *<0.01$

559 Table 1. Description of the quantified behavioral variables and their transformations.

560 Table 2. PCA loadings of behaviors.

561 Table 3. Summary of the repeatability tests on the three principal components extracted from 562 behavioral variables. 


\section{Figure 1}

Behavioral testing box $(40 \times 25 \times 25 \mathrm{~cm})$ used for the mirror-image stimulation study in Microcebus murinus.

Three zones were delimited to quantify the behavioral reaction. The sidewall of zone 1 is either transparent facing the other box, a mirror $(20 \times 20 \mathrm{~cm})$, or an opaque board, depending on the test context. The animal enters the test area in zone 3.

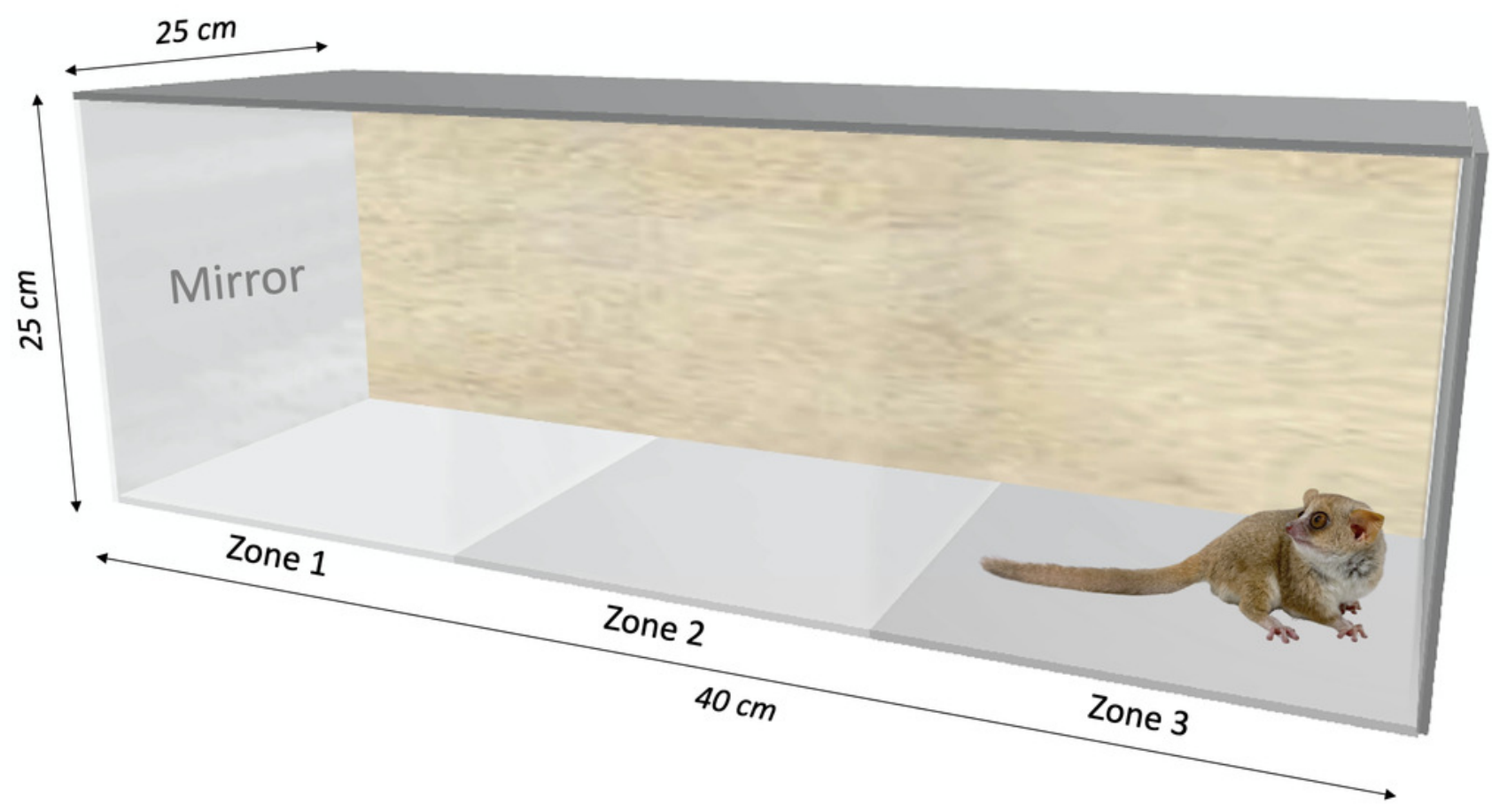




\section{Figure 2}

Boxplots of principal component analyses run on behavioral variables quantifying reaction to mirror-image stimulations in female $(n=6)$ and male $(n=6)$ mouse lemurs (Microcebus murinus).

The figure shows (A) Principal Component (PC) 1 related to "activity", (B) PC2 which can be described as an "approach score", and (C) PC3 which can be described as an "anxiety score", separated by sex and test context. The contexts were: (Mir) mirror-image stimulation, the mirror visible; (OF) a neutral control with an opaque and lateral panel; (T) a positive control context (i.e., facing a conspecific). Each point represents an individual. The middle line of the boxplot represents the median. The lower and upper hinges correspond to the first and third quartiles (the 25th and 75th percentiles). The upper and lower whisker extends from the hinge to the largest and smallest value no further than $1.5 *$ IQR from the hinge (where IQR is the inter-quartile range, or distance between the first and third quartiles). $P$ value $*<0.05 ; * *<0.01$ 

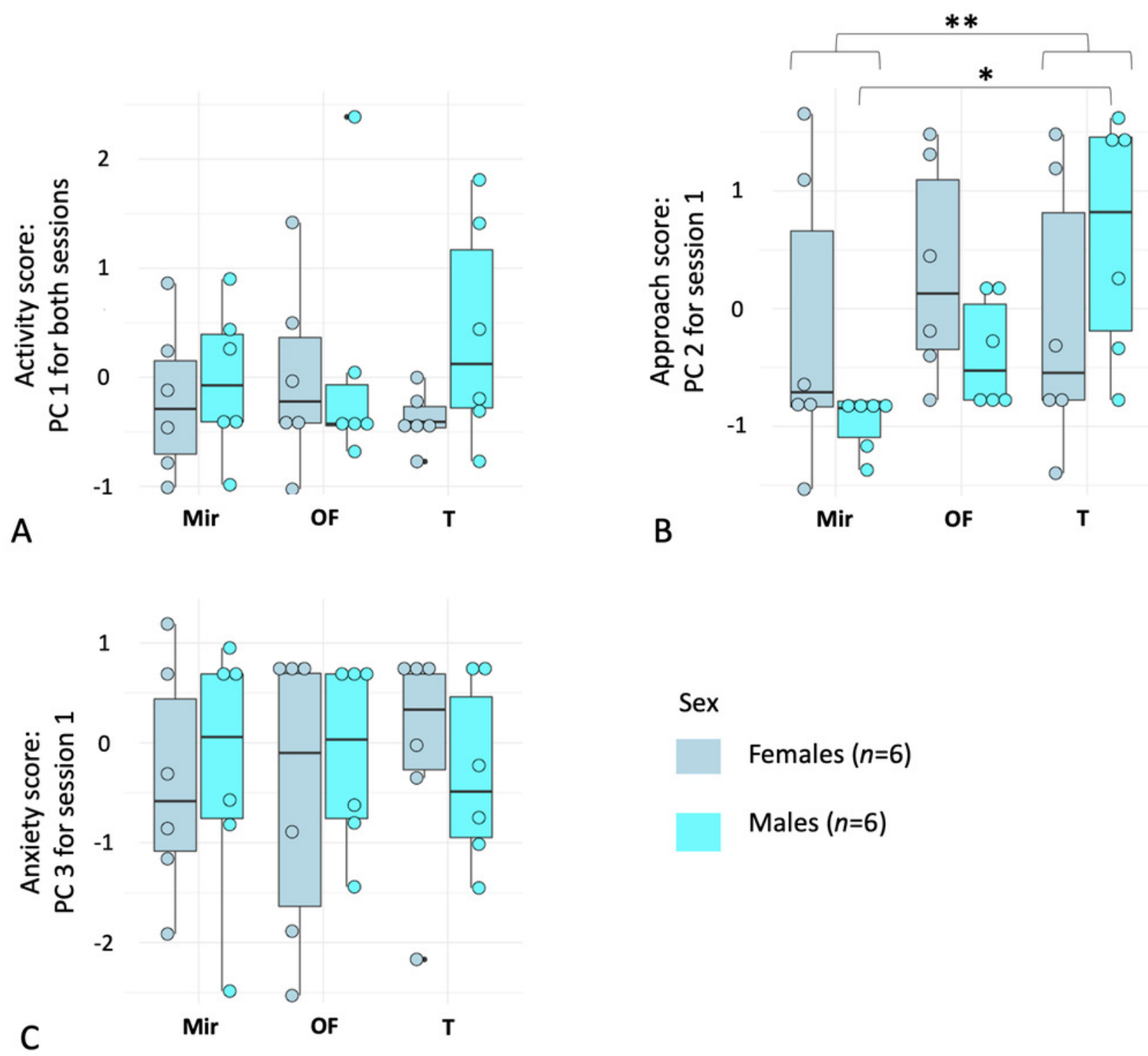

Sex

Females $(n=6)$

Males $(n=6)$ 


\section{Table 1 (on next page)}

Description of the quantified behavioral variables and their transformations 


\begin{tabular}{|l|l|}
\hline \multicolumn{1}{|c|}{ Behavioral variable } & \multicolumn{1}{|c|}{ Description } \\
\hline Latency to first movement & $\begin{array}{l}\text { Time elapsed before crossing from zone 3 } \\
\text { to zone 2. }\end{array}$ \\
\hline Number of changes of zones & The number of times moving from one \\
zone to another.
\end{tabular}

2 
Table 2 (on next page)

PCA loadings of behaviors. 
1

\begin{tabular}{|l|c|c|c|}
\hline & PC1 & PC2 & PC3 \\
\hline Latency to First movement & -0.38 & & $\mathbf{0 . 9 0}$ \\
\hline Number of changes of zone & $\mathbf{0 . 8 7}$ & & -0.37 \\
\hline Time spent rearing & $\mathbf{0 . 7 8}$ & & -0.33 \\
\hline Latency to approach & -0.43 & & $\mathbf{0 . 8 8}$ \\
\hline Number of approaches & $\mathbf{0 . 8 7}$ & & -0.38 \\
\hline Number of mirror contact & $\mathbf{0 . 7 8}$ & $\mathbf{0 . 2 9}$ & -0.19 \\
\hline Time spent in the mirror cell & & $\mathbf{0 . 9 7}$ & \\
\hline Number of rearing & $\mathbf{0 . 8 5}$ & & -0.375 \\
\hline
\end{tabular}

2 Variables loading strong on each axis are indicated in bold 


\section{Table 3(on next page)}

Summary of the repeatability tests on the three principal components extracted from behavioral variables. 
1

\begin{tabular}{|l|c|c|c|c|}
\hline & $\mathrm{R}$ & $\mathrm{SE}$ & $\mathrm{CI}$ & $\mathrm{P}$ \\
\hline PC1 & 0.288 & 0.128 & {$[0.023,0.523]$} & 0.003 \\
\hline PC2 & 0.12 & 0.102 & {$[0,0.361]$} & 0.085 \\
\hline PC3 & 0.034 & 0.068 & {$[0,0.216]$} & 0.296 \\
\hline
\end{tabular}

2

3 